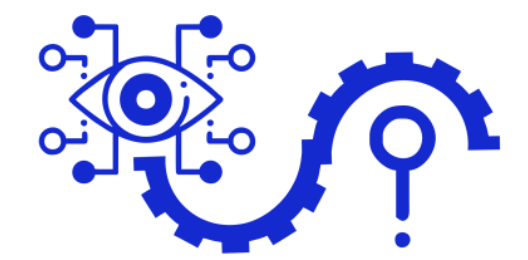

REVISTA DE INVESTIGACIÓN E INNOVACIÓN

\title{
Apuntes sobre la identidad docente en la educación superior como guía para la calidad y gestión académica
}

Notes on the teaching identity in higher education as a guide for academic quality and management

\begin{abstract}
Autores
Aguirre Vilchez, Kenia

Universidad Nacional de Huancavelica

Ramos Serrano, Rúsbel Freddy ORCID

Universidad Nacional de Huancavelica

Arias Sánchez, Raúl Eleazar ORCID

Universidad Nacional de Huancavelica
\end{abstract}

\section{RESUMEN}

Este ensayo es una breve e inicial revisión de la identidad que los docentes de educación superior cultivan y mantienen como soporte para mejorar las condiciones de calidad académica y moral. En ese sentido, esta reflexión pretende mostrar dos escenarios de actuación, el primero relacionado con lo "formal" y el otro con lo "real", dando a conocer que ambos convergen para dar paso a acciones de trabajo interpersonal y proyección externa de los agentes educativos. por el bien de una institución. El presente estudio utilizó como método principal el método etnográfico para la recolección de información cualitativa. Nuestros entrevistados señalaron que la identidad institucional puede contribuir significativamente a los procesos organizacionales. Finalmente, nuestra investigación concluyó que la identidad docente en la educación superior sirve como guía para la calidad y gestión académica.

Palabras clave: Identidad; calidad académica; educación superior.

\begin{abstract}
This essay is a brief and initial review of the identity that teachers in higher education cultivate and maintain as a support to improve the conditions of academic and moral quality. In this sense, this reflection aims to show two performance scenarios, the first related to the "formal" and the other to the "real", making it known that both converge to give way to interpersonal work actions and external projection of the educational agents for the good of an institution. The present study used the ethnographic method for the collection of qualitative information as the main method. Our interviewees concluded that institutional identity can contribute significantly to organizational processes. Finally, our research concluded that the teaching identity in higher education serves as a guide for academic quality and management.
\end{abstract}

Keywords: Identity; quality of education; higher education. 


\section{GnosisWisdom}

\section{INTRODUCCIÓN}

Hoy en día, sabemos que el mundo viene atravesando importantes cambios en sus diversas estructuras e instituciones sociales, políticas, educativas, económicas y culturales a causa de la pandemia COVID-19, la cual sin duda ha evidenciado nuestras dificultades y límites como país, sin embargo, también nos ha permitido generar algunas soluciones innovadoras que de todas formas necesitan impulso y promoción. Así, dentro de esta coyuntura, el escenario del sistema de educación superior universitaria no ha sido ajeno y ha presentado algunos cambios y quiebres importantes en el acondicionamiento de un estilo diferente dentro del asunto de enseñanza-aprendizaje y el compromiso de los agentes educativos de manera virtual, evidenciando con ello una nueva identidad docente marcada en algunas situaciones y sigilosa en otras. De esta manera, concordamos con Cevallos (2014) cuando indica que,

"La preocupación fundamental hoy de la evaluación y regulación de la calidad mayor parte de los países, es mantenimiento, mejoramiento y garantía de propósitos y procedimientos, que es la calidad de la educación superior, imposible considerarlas separadamente. Se haciéndose enormes esfuerzos por lograr acredita conforme a un proceso de su adecuada definición, medición y evaluación y de seguimiento, para disponer evaluación." (p. 3).

Asimismo, consideramos que esa tan añorada calidad podría ser posible cuando exista una relación horizontal y asertiva entre todos los involucrados del escenario educativo a nivel formal y familiar.

De igual forma, este esfuerzo y ejercicio académico podría cimentarse desde la percepción de identidad del docente sobre su entorno y querer hacer de éste un escenario de bienestar y oportunidad. En este sentido, este ensayo pretende establecer algunas reflexiones en cuanto al tema de identidad docente en un contexto universitario y cómo ésta permitiría desarrollar una mejor gestión educativa.

Para Lagarde (2000) la categoría identidad posee varios matices como:

“...la identidad asignada, la identidad aprendida, la identidad internalizada que constituye la autoidentidad. La identidad siempre está en proceso constructivo, no es estática ni coherente, no se corresponde mecánicamente con los estereotipos. Cada persona reacciona de manera creativa al resolver su vida, y al resolverse, elabora los contenidos asignados a partir de su experiencia, sus anhelos y sus deseos sobre sí misma. Más allá de las ideologías naturalistas y fosilizadoras, los cambios de identidad son una constante a lo largo de la vida. Sus transformaciones cualitativas ocurren en procesos de crisis. Por ello, la identidad se define por semejanza o diferencia en cuanto a los referentes simbólicos y ejemplares. Cada quien es semejante y diferente. Finalmente, cada quien crea su propia versión identitaria: es única o único.” (p.61).

De lo citado cabe indicar que cada persona construye su propio sentido de pertenencia hacia su grupo y/o comunidad. De igual forma, las instituciones, al igual que los individuos, construyen a través del tiempo una identidad colectiva, que nace de los referentes culturales y económicos de sus actores inmediatos directos e indirectos, que somos en último término los 


\section{GnasisWisdom}

ciudadanos o quienes laboramos dentro de ellas (Mercado \& Hernández, 2010).

Las instituciones académicas y formativas, más allá de ser el espacio de producción intelectual y tecnológica son espacios que generan desarrollo cuando persisten en los valores que las caracterizan con miras a generar un salto positivo cualitativo y cuantitativo. Esto es posible, a nuestro criterio, por el compromiso inherente dentro de la condición de docente o maestro.

\section{Sobre la identidad docente}

Ante este escenario, podemos decir que, la identidad del docente con su institución permite identificar elementos constructivos y disruptivos orientados al logro de objetivos, metas y calidad académica institucional. Sin embargo, no siempre se recibe el apoyo o incentivo necesario, al respecto López \& de Serdio (2011) indican que,

"La falta de criterios para organizar de manera específica la promoción y la valoración de la investigación en Ciencias Sociales y las Humanidades y la promoción de una investigación basada en la resolución de problemas, productiva y validada en el mercado ha generado (y sigue generando) una situación de incomodidad...” (p. 82).

Es cierto que el problema presentado genera malestar ante tanta exigencia que en muchas ocasiones tampoco es asumida por quienes las exigen. Así, sale a la luz el tema de identidad, los autores citados refirieron al respecto que,

"Los cambios en la vida profesional de los docentes universitarios responden a transformaciones contextuales, políticas, económicas, sociales y tecnológicas que instrumentalizadas a través de diversas reformas y reestructuraciones han provocado un profundo cambio en la naturaleza del trabajo académico y, consecuentemente, en la identidad de sus protagonistas." (p. 84).

Esto reflejaría de forma empírica que habría docentes comprometidos con su institución al inicio de su labor, sin embargo, poco a poco ese nivel de significancia identitaria iría cambiando y disminuyendo al presentarse imponderables cada vez menos predecibles y confiables.

Por otro lado, Monereo \& Domínguez (2014) tras realizar una investigación vinculada al tema de identidad y comportamiento docente concluyeron de sus entrevistados y encuestados que,

“...la docencia universitaria supone una gran responsabilidad, en tanto influye decisivamente en la formación de profesionales que ejercerán funciones en el mundo laboral y social. Al mismo tiempo dicho rol constituye, para la mayoría de los entrevistados, una elección vocacional que conlleva satisfacción personal y profesional, apreciándose en todos los casos una trayectoria de continua formación y desarrollo. En su quehacer cotidiano, cobra especial importancia la formación continua y la actualización permanente, tanto en aspectos pedagógicos como en la necesidad de estar al día en sus respectivas disciplinas." (p. 91).

Entonces podemos asumir que, una característica que compartimos muchos docentes universitarios dentro de la complejidad y matiz de nuestras identidades, es el sentido extenso de la gran tarea de formar hombres y mujeres, los cuales se enfrentarán a una sociedad salvaje y quizá, a la vez, sean más adelante quienes velen por nuestro propio futuro. 


\section{GnasisWisdom}

Otra característica también es señalada por los citados, de la cual estamos totalmente de acuerdo por la evidencia fáctica de nuestro actuar diario,

"El buen profesor es también descrito como un profesional preocupado por la innovación metodológica docente, que se embarca en actividades de mejora educativa y reflexiona acerca de sus aciertos y errores en este proceso. Aquí aparecen claramente elementos de carácter emocional que deben presidir las actitudes de un buen docente, como tener pasión por lo que se hace..." (Monereo \& Domínguez, 2014 p. 92).

\section{Sobre la pasión del docente}

Así, tenemos una segunda característica que forja nuestra identidad docente, y es la pasión, esa forma perseverante y dinámica para crear escenarios y situaciones llamativas para los estudiantes, empleando toda su capacidad inventiva e ingenio para lograr transmitir información y conocimiento a la vez.

Sin duda estas fortalezas al orientarlas y alentarlas de manera adecuada lograrían un mejor desempeño e identificación con la institución, sin embargo, a pesar de que muchas autoridades asumen esta "teoría" muchas veces no se ve reflejada en la práctica, por lo que también existen situaciones nada agradables.

"Los sentimientos negativos, manifestados frente al rol docente, apuntan principalmente a la escasa valoración institucional que tiene este rol a pesar del alto nivel de preparación y trabajo que supone. Argumentan que la investigación es mucho más valorada que la docencia, lo que claramente beneficia a aquellos que dedican mucho tiempo a investigar y publicar, en detrimento de la calidad de sus clases. Un segundo factor que dispara sentimientos negativos guarda relación con lo que algunos consideran inmadurez de los estudiantes al no mostrar un suficiente nivel de auto-exigencia en sus tareas académicas o al no valorar todo el esfuerzo que el profesor invierte en conseguir una buena docencia." (Monereo \& Domínguez, 2014 p. 96).

Estas dos variables delimitadas, la escasa valoración institucional y la inmadurez de los estudiantes tendrían que ser el foco de atención para generar también políticas educativas que permitan a la institución superarlas en el mediano y largo plazo. Un ejemplo de esta poca valoración es la referencia que nos hizo un colega quién prefirió mantenerse en el anonimato, pero estuvo deseoso que su percepción fuese registrada:

“...yo me siento identificado con nuestra universidad, sé que soy contratado, pero siempre trato de cumplir con lo que me encargan...todo está bien, pero el detalle de esta universidad (Universidad Nacional de Huancavelica) es que no nos pagan a los contratados que entramos a trabajar desde marzo de este año (2021) ...firmemente creo que eso es un abuso y que vulnera nuestro derecho y sobre todo a veces hace que se pierda el deseo de apoyar a la universidad...¿ide qué se vive? Si no hay sueldo...

Como hemos podido apreciar, no siempre las cosas marchan bien, y creemos que esto se debe a que nos hacen faltan mejores formas de gestión institucional, así como también, asertividad en la valoración del docente universitario quien presta sus servicios como cualquier otro trabajador por un salario, sin embargo, a diferencia de muchos, la pasión y responsabilidad sobrepasa la situación individual, ya que el producto 


\section{GnasisWisdom}

que forje (estudiantes egresados) servirá a un bien mayor y colectivo. Es decir, a pesar de todo lo actuado y percibo tanto de forma negativa como positiva van formando y alineado una identidad docente, al respecto Ruibal, Valenzuela, Félix \& Lúgigo (2013) sugieren que,

"Este resumen del perfil identitario, claramente se ha construido en los campos socioinstitucionales de la disciplina, la profesión, el aula, y ligeramente en la investigación, sin dejar de lado las cuestiones afectivas y emocionales. Esta identidad encadenada al rol tradicional de la docencia es un reflejo de esos procesos de diferenciación del trabajo académico a otras labores, esta diferenciación apoya la idea de una autonomía y una autoorganización." (p. 93).

Esta dialéctica entre "lo bueno" y "lo malo" trae como consecuencia que nuestra identidad toma caminos que al ser estimulados puedan no dar marcha atrás. Entonces si asumimos esta premisa, podríamos indicar que un buen ambiente de trabajo genera un buen desempeño en él, a este cometido podemos sumar las apreciaciones de Sáez, Fraile \& Loroño (2015) quienes señalan que,

Esta dialéctica entre "lo bueno" y "lo malo" trae como consecuencia que nuestra identidad toma caminos que al ser estimulados puedan no dar marcha atrás. Entonces si asumimos esta premisa, podríamos indicar que un buen ambiente de trabajo genera un buen desempeño en él, a este cometido podemos sumar las apreciaciones de Sáez, Fraile \& Loroño (2015) quienes señalan que,

En la misma línea de los citados, Fitzmaurice (2013) incorpora una dimensión moral y de valores que suponen también parte de la estructura de la identidad docente, que a pesar de las "negatividades" se mantiene y logra sobreponerse ante la "adversidad institucional", teniendo así el objetivo claro de lograr la calidad académica, sobre lo señalado Martín, Conde \& Mayor (2014) dicen que,

"Este indicador nos alerta sobre la necesidad de invertir tiempo y esfuerzo en el diseño y puesta en marcha de programas formativos dirigidos a la formación inicial del profesorado universitario. Además, ha de considerarse que el desarrollo profesional de los docentes, no es algo exclusivamente dependiente del adecuado diseño, en cuanto a metodología o contenidos, de los programas dedicados a formarlos. Este desarrollo depende en gran medida de la institución en la que los profesores desempeñan su rol profesional. Su desarrollo se encuentra ligado al desarrollo del centro." (p. 143).

A la luz de esta premisa, si una institución proporciona condiciones favorables, la identidad del docente se mostrará sólida y generar espacios de crecimiento y desarrollo. Fuentes (2012) con un criterio más incisivo señala que,

"Ante la posibilidad de acceder a distintos escenarios configurados por las propias tareas educativas, el maestro debe enfrentar la incertidumbre que su trabajo implica. Para ello, cuenta con el bagaje de su formación disciplinar, el cual le provee de los elementos necesarios para atender los primeros retos que su práctica docente y educativa le asignan. Aquí se ubica la génesis de su segunda identidad, aquella que le permite percibirse como parte de un grupo, a cuya consonancia se 


\section{GnasisWisdom}

debe (la primera es aquella que deviene de la educación recibida en el seno familiar y que le define como persona). Bajo los rituales, saberes y creencias que alimentan dicha integración grupal, el maestro, en calidad de profesional, logra vencer los obstáculos que el ejercicio docente le asigna, lejos de una identidad congruente con su nuevo papel. La única identidad que reconoce y acepta es la de su gremio de profesionistas, en muchas ocasiones representado institucionalmente por un pequeño grupo de maestros que llegan a coincidir dentro de los planteles educativos." (p. 32).

Sin duda, este reflejo académico hace que nos acerquemos a la dinámica de subjetividad del docente y a la diversidad de percepciones inscritas dentro de la misma. Por un lado, al configurarse el sentido de pertenencia hacemos todo lo posible para que "salgan bien las cosas", y por otro, si factores externos debilitan "ese sentido" nos veremos en una disyuntiva asociada a un desempeño únicamente instrumentalista y mecánico, los cuales sería poco productivos en el medio. Este panorama también es entendido por García (2010) quien manifiesta que,

"Hay que entender el concepto de identidad docente como una realidad que evoluciona y se desarrolla tanto personal como colectivamente. La identidad no es algo que se posea sino algo que se desarrolla a lo largo de la vida. La identidad no es un atributo fijo para una persona, sino que es un fenómeno relacional. El desarrollo de la identidad ocurre en el terreno de lo intersubjetivo y se caracteriza como un proceso evolutivo, un proceso de interpretación de uno mismo como persona dentro de un determinado contexto." (p. 19).

Así, el ideal sería que en cada espacio educativo se nos proporcione las herramientas necesarias para un adecuado desempeño docente, aunque también sería algo utópico en la práctica, ya que, si consideramos una ontología en la construcción de conocimiento, ciencia e identidad, las cosas más relevantes para un escenario significativo serían las exigencias y "sinsabores".

Es decir, manejar el paradigma de que después de cada conflicto, éste se transformaría en una oportunidad para nosotros y para nuestra institución educativa.

\section{METODOLOGÍA}

El presente estudio utilizó como método principal el método etnográfico, que en palabras de Giddens (2007) este se constituye como

“...el estudio directo de personas y grupos durante un período determinado, utilizando la observación participante o entrevistas para conocer su comportamiento social, registrando una imagen realista y fiel del grupo estudiado; el trabajo de campo resulta ser una herramienta fundamental". (p. 27).

Esta metodología cualitativa contempló la recolección de información in situ, a través de entrevistas y la observación participante, las cuales nos permitieron lograr un acercamiento con los involucrados sobre sus percepciones respecto al tema planteado.

- Alcance de la investigación: Nuestra investigación abarcó el escenario de la Universidad Nacional de Huancavelica.

- Diseño de la investigación: Se utilizó un diseño descriptivo, propio de la investigación cualitativa. 


\section{GnosisWisdom}

- Procedimiento de Investigación: Esta investigación tomó como referencia las entrevistas estructuradas realizadas a 15 docentes contratados de diversas Facultades de la Universidad Nacional de Huancavelica. Las entrevistas fueron transcritas y grabadas con el consentimiento de los participantes, asimismo, algunos de ellos decidieron dar sus opiniones de forma anónima, hecho que respetamos en todo momento.

- Recopilación de datos y análisis de datos: La recolección de información se realizó a través de entrevistas estructuradas y una encuesta, las cuales fueron validadas a través de un juicio de expertos.

\section{RESULTADOS}

A continuación, presentamos algunos extractos de entrevistas que indican en lo "real" las percepciones de algunos docentes de educación superior sobre lo planteado en el presente documento:

- “...la verdad es que estoy cansada de esta situación, como contratada hay que hacer de todo para complacer al jefe, ...sólo lo hago porque quiero a mi universidad...es mi alma mater..."

- “...a veces me cuesta trabajar en la oficina, no hay nada... tengo que comprar mis propios papeles y sacar mis copias para los alumnos...pero bueno...lo hago con gusto, por mis muchachos..."
- “...te cuento que tengo varias cosas en la cabeza, deudas del banco, mi mujer (risas)... pero este lugar (la universidad) me ha dado todo... así me traten mal (risas) estaré aquí apoyando..."

- “...la verdad todo lo hago por mi UNH, sé que a veces el decano me mira mal, pero no me importa, primero es mi camiseta porque estudié acá...”

- “...no te miento cuando te digo que a pesar del sueldito que tenemos, me siento bien...la docencia es otra cosita...es ser responsable del futuro que daremos a nuestros hijos...no se gana, pero se goza mi querido profe..."

Sobre lo expuesto, podemos señalar que la docencia representa para muchas personas una gran responsabilidad a la vez que, se convierte en una pasión por el servicio a pesar de las condiciones adversas que se puedan presentar en términos institucionales.

\section{DISCUSIÓN}

Creemos que nuestra pesquisa tomó una muestra significativa de la percepción de la identidad institucional de los docentes, la cual necesita fortalecerse ya que al hacerlo podría generar mejores espacios de trabajo y productividad dentro de la universidad. Sin embargo, a pesar de la literatura y las políticas educativas existentes, estamos lejos de encaminar las mejores prácticas para el buen desempeño de nuestros docentes y con ello mejorar la calidad educativa, por lo que es necesario reestructurar la gestión sectorial, mejorar los salarios y los ambientes laborales, entre otros. 


\section{GnasisWisdom}

\section{CONCLUSIONES}

Finalmente, deseamos expresar en este breve ensayo que, a pesar de la diversidad humana y social que alberga nuestro entorno, hemos encontrado la fortaleza de la identidad y que, en esencia debería de ser valorada ya que es la única fuente que puede servir de agente de cambio para nuestro presente y futuro, para nosotros y para quienes vendrán después de nosotros, y su manifestación material se ve reflejada en la figura del maestro o docente. Éste en su multidimensionalidad va construyendo a lo largo de su experiencia íntima y colectiva una identidad que, de ser retadora, encaminaría procesos de alta calidad y competitividad, así como también, escenarios de innovación y desarrollo. Queda sin duda una tarea de acercamiento más institucional y un reto dentro de las políticas educativas a largo plazo.

\section{REFERENCIAS}

[1] Cevallos, D. (2014). La calidad educativa en la realidad universitaria peruana frente al contexto latinoamericano. Revista de la Universidad Católica Santo Toribio de Mogrovejo Flumen. 7 (1): 3-8

[2] Fitzmaurice, M. (2013). Constructing professional identity as a new academic: a moral endeavour. Studies in Higher Education. Vol. 38. No4: 613-622

[3] Fuentes, M. (2012). Identidad docente y exigencia académica: encuentros y desencuentros con la realidad social. Perspectiva Educacional, Formación de Profesores, 51(1), 29-44.

[4] García, C. (2010). La identidad docente: constantes y desafíos. Revista Interamericana de Investigación, Educación y Pedagogía, 3(1), 15-42.
[5] Giddens, A. (2007). Sociología. Madrid, España: Alianza Editorial.

[6] López, A. \& de Serdio, A. (2011). Sujeto a reforma: la transformación en la identidad docente en la educación superior. Revista de Sociología de la Educación-RASE, 4(1), 73-88.

[7] Martín, Á., Conde, J., \& Mayor, C. (2014). La identidad profesional docente del profesorado novel universitario. REDU. Revista de docencia universitaria, 12(4), 141-160.

[8] Mercado, A. \& Hernández, A. (2010). El proceso de construcción de la identidad colectiva. Convergencia, 17(53), 229-251.

[9] Monereo, C. \& Domínguez, C. (2014). La identidad docente de los profesores universitarios competentes. Educación XX1, vol. 17, núm. 2, julio-diciembre, 2014, pp. 83-104

[10] Ruibal, R., Valenzuela, B. Félix, G. \& Lúgigo, M. (2013). La identidad docente, la política y gobernabilidad en educación superior. European Scientific Journal, 9(2).

[11] Sáez, I., Fraile, C. \& Loroño, M. (2015). La identidad profesional docente como clave para el cambio en la educación superior. Opción, 31(5), 51-74. 